\title{
Estimation-based Disturbance Rejection in Control for Limit Cycle Generation on Inertia wheel Inverted Pendulum Testbed
}

\author{
S. Andary, A. Chemori and S. Krut
}

\begin{abstract}
This paper deals with constant disturbances rejection in limit cycle tracking for an underactuated mechanical system. The feedback controler presented in [1] is enhanced to handle constant disturbances by using online iterative estimation of an equivalent disturbance which is easily compensated by adding the estimated value to the output of the system. The effectiveness of the proposed method is demonstrated through real-time experiments on an inertia wheel inverted pendulum. Constant disturbances are introduced either as a weight asymmetrically fixed to the pendulum body, or by the use of a bad calibrated sensor.
\end{abstract}

\section{INTRODUCTION}

Disturbance rejection has widely been considered in feedback control design. The exogenous disturbance signals are often modeled as unknown deterministic signals with known signal generating dynamics. For linear systems with linear disturbance dynamics, this problem has been studied in [3]. The concept of Internal Model Principle (IMP) has been initially proposed in [5] showing that it is necessary to place the disturbance dynamics in the feedback control loop in order to compensate them through asymptotic tracking. The proposed concept of IMP has also been used in repetitive control [13] and Integral control. In [8], a linear-disturbance observer based approach was proposed for disturbance rejection, and has widely been used in industrial applications [4] [9] mostly for linear systems.

Other researchers have been interested in disturbance rejection in the case of nonlinear systems with linear and in some cases slightly nonlinear disturbance dynamics. In [2], the authors propose an extension of the method proposed in [8] to fully actuated non-linear systems. The proposed extended method consists in estimating external or internal disturbances in order to compensate them. In [7] the problem of global output regulation was addressed in the case of an exogenous disturbance with bounded initial conditions. In [6] a $k^{\text {th }}$-order robust nonlinear servomechanism design was proposed, for which the necessary and sufficient conditions for existence have been discussed. In [14] the problem of chaotic exogenous signals rejection with known nonlinear dynamics is addressed. The proposed feedback controllers are based on IMP concept and predictive internal model control.

S. Andary, A. Chemori, and S. Krut are with LIRMM, Univ Montpellier 2 - CNRS, 161 rue Ada, 34392 Montpellier, France andary@lirmm.fr
This paper addresses the problem of constant disturbances rejection in periodic reference trajectories tracking for a nonlinear underactuated mechanical system, namely the inertia wheel inverted pendulum, through an extension of the control scheme proposed in [1]. Based upon partial feedback linearization and reference trajectories optimization, the original approach deals with stable limit cycle generation for underactuated mechanical systems with application to the inertia wheel inverted pendulum. Limit cycles are generated on both actuated and unactuated parts of the system. Furthermore, it has been shown that the controller is robust towards punctual disturbances. Indeed it is able to reject them and steer the system asymptotically to the limit cycle. The present work is the continuity of that proposed in [1], the aim is to show the limitations of the original proposed controller to deal with persistant disturbances and to propose an extension to enhance its robustness towards them.

In the case of the proposed application, i.e. inertia wheel inverted pendulum, persistant disturbances are phenomena coming from different sources, such as inclinometer offset $^{1}$, pendulum-mass repartition asymmetry or model uncertainties. These disturbances induce some troubles like errors in trajectory tracking, instability of the limit cycle or decrease of the capacity of the controller to reject punctual disturbances.

To deal with the problem of persistant-disturbances rejection, the proposed technique is based on the controller proposed in [1] and an iterative online estimation of the disturbance which is chosen to be easily compensated in control loop using an introduced $\delta$-parameter.

The organization of this paper is as follow: in Section II, a brief description of the plant and its dynamics is presented. Section III presents briefly the paremeterizedreference trajectories. The main result of the paper is presented in section IV, where the addressed problem is discussed as well as the proposed solution. Experimental results are presented and argued in section $\mathrm{V}$. The paper ends with some concluding remarks.

\section{Testbed Description and Dynamics}

The mechanical system studied in this paper is the inertia wheel inverted pendulum shown in Figure 1. It

\footnotetext{
${ }^{1}$ The inclinometer is the sensor used to measure the angle of the pendulum with respect to the vertical (cf. Fig. 1), it gives measures with offset whenever it isn't straightly fixed.
} 
consists of an inverted pendulum with a rotating inertia wheel. The pendulum axis joint is not actuated, while the inertia wheel joint is actuated by a DC motor. The system has two degrees of freedom and only one actuator, therefore it belongs to the class of nonlinear underactuated mechanical systems. Figure 2 displays a schematic view of the mechanical structure of the system explaining its functioning principle. In the sequel, the

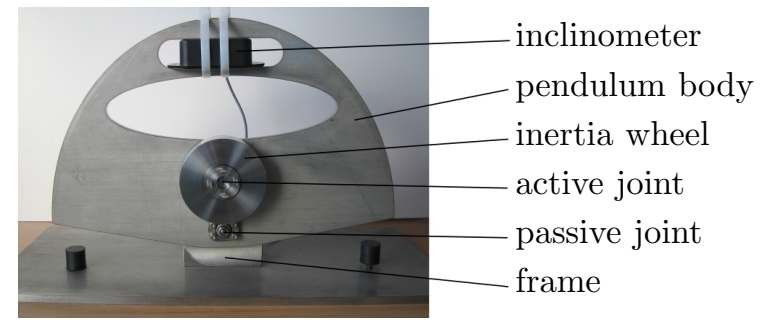

Fig. 1. View of the inertia wheel inverted pendulum testbed

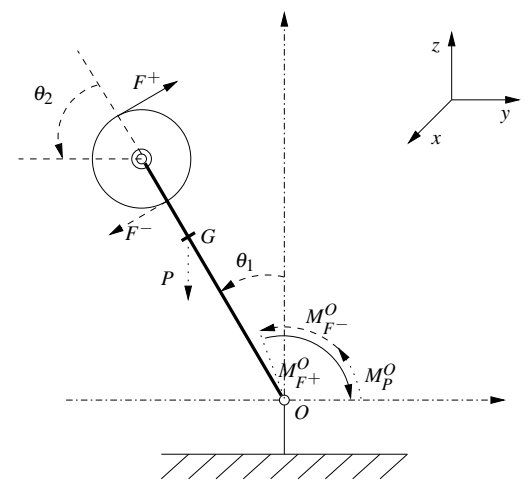

Fig. 2. Mechanical principle: the inertia wheel is assumed to be equivalent to two punctual masses situated at the acting points of $F^{+}$and $F^{-}$. To straighten up the pendulum, the torque acting at $O$ generated by $F^{+}$must be greater than that generated by both gravity and $F^{-}$forces.

nonlinear dynamic model of the system is presented.

\section{A. Dynamic Modelling of the plant}

The non linear dynamic model of the system [10] is described by the following equations ${ }^{2}$ :

$$
\begin{aligned}
& \ddot{\theta}_{1}=\frac{1}{i_{1}}\left(C_{1}-C_{2}+\overline{m l} g \sin \theta_{1}\right) \\
& \ddot{\theta}_{2}=\frac{1}{i_{1} i_{2}}\left[\left(i_{1}+i_{2}\right) C_{2}-i_{2} C_{1}-i_{2} \overline{m l} g \sin \theta_{1}\right]
\end{aligned}
$$

where $\theta_{1}, \theta_{2}$ are respectively angular positions of the pendulum and the inertia wheel. $i_{1}, i_{2}$ are respectively their moments of inertia. $C_{1}$ is the perturbation torque applied to the pendulum, while $C_{2}$ is the torque generated by the actuator acting on the inertia wheel. $C_{1}$ is considered to be null in the design of the control approach. $\overline{m l}=m_{1} l_{1}+m_{2} l_{2}$ with $m_{1}$ and $m_{2}$ being the masses of the pendulum and the inertia wheel. $l_{1}, l_{2}$ are distances from origin $O$ to gravity center of the pendulum and the rotating mass.

\footnotetext{
${ }^{2}$ Note that this dynamic model is obtained by application of the Lagrange formulation.
}

\section{Parameterized Reference Trajectories Optimization}

The proposed controller (cf. next section) is based on partial feedback linearization, which is performed with respect to the unactuated coordinate $\theta_{1}$. Then it would be necessary to generate reference trajectories (i.e. $\theta_{1}^{r}$ : desired position, $\dot{\theta}_{1}^{r}$ : desired velocity, and $\ddot{\theta}_{1}^{r}$ : desired acceleration).

The proposed reference trajectory ${ }^{3}$ on position is a polynomial $p$-parameterized trajectory, where the parameter $p$ should be computed, in order to be fully defined. An optimization problem is to be resolved at the beginning of each oscillation-cycle, of period $\tau$, to compute the parameter $p$ allowing the pendulum body to spend more time on either side of the equilibrium point. The objective is to minimize as much as possible the velocity of the inertia wheel at the end of each cycle using a quadratic function in the performance index of the optimization problem. A typical normalized position reference-trajectory is that plotted in figure 3 for a half of a period of the oscillationcycle.

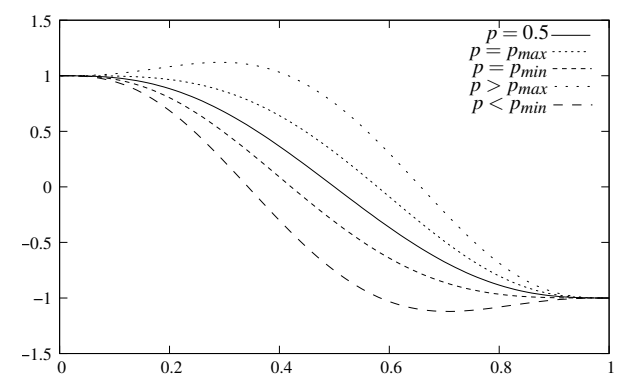

Fig. 3. Normalized reference trajectory during half a period for different values of $p\left(p_{\min }=0.4214, p_{\max }=0.5786\right)$. $p_{\min }$ and $p_{\max }$ are extreme values of $p$, such as the p-parameterized trajectory remains within the interval $[-1,1]$

The reference trajectories on velocity and acceleration are obtained through successive time derivatives of the position's reference trajectory.

\section{Addressed Problem and Proposed Solution}

Consider the control approach proposed in [1] for limit cycles generation. The control law is based on the concept of partial feedback linearization [12], [11] which results in the following:

$$
U=i_{1}\left[\frac{\overline{m l} g}{i_{1}} \sin \left(\theta_{1}\right)-\ddot{\theta}_{1}^{r}+k_{p}\left(\theta_{1}-\theta_{1}^{r}\right)+k_{v}\left(\dot{\theta}_{1}-\dot{\theta}_{1}^{r}\right)\right]
$$

where $k_{p}$ and $k_{v}$ are respectively the proportional and derivative gains and are used to tune the dynamics of the closed loop system. $\theta_{1}^{r}, \dot{\theta}_{1}^{r}$ and $\ddot{\theta}_{1}^{r}$ are, respectively, reference trajectories for position, velocity and acceleration of the pendulum body. Given the system's dynamic model and the control law, we can express the internal

\footnotetext{
${ }^{3}$ Since the objective is to generate stable limit cycles, the proposed reference trajectories are periodic.
} 
dynamics of the system by injecting (3) in (2) with $C_{1}=0$ and $C_{2}=U$, one obtains:

$$
\ddot{\theta}_{2}=-\frac{i_{1}+i_{2}}{i_{2}}\left[\ddot{\theta}_{1}^{r}-k_{v}\left(\dot{\theta}_{1}-\dot{\theta}_{1}^{r}\right)-k_{p}\left(\theta_{1}-\theta_{1}^{r}\right)\right]+\frac{\overline{m l} g}{i_{2}} \sin \theta_{1}
$$

The state vector of the internal dynamics includes inertia-wheel's position and velocity. Since the system is non minimum phase, then the internal dynamics is unstable. However, as the inertia wheel is not limited by mechanical stops when rotating around its axis, its angular position does not matter. Consequently it is worth interesting only in its velocity.

In case of a persistant and constant disturbance, a nonzero inertia wheel velocity is obtained, at the end of each cycle, because the controller does not take into account the external disturbance. Indeed, the optimization is performed given a nominal model of the system, that does not take into account the external perturbation, so the chosen parameter does not achieve to bring the inertia wheel velocity cycle around zero and the limit cycles are no longer stable.

\section{A. Proposed Solution}

To overcome the problem of the external persistant disturbances, the controller has to compensate for them. For that, let us introduce a new parameter $\delta$ representing the disturbance in the model. The basic idea comes from the fact that a constant disturbance applied to the system during tracking of reference trajectories results in an offset in the instable equilibrium point, which affect the oscillation cycle of the pendulum. This offset causes a divergent oscillation of the inertia wheel velocity. A simple way of shifting back the oscillation of the pendulum as well as the inertia wheel velocity around zero is to translate the trajectory in one side of the equilibrium. We introduce then the parameter $\delta$ as follows:

$$
\theta_{1}^{*}=\theta_{1}+\delta
$$

where $\theta_{1}^{*}$ is the disturbed pendulum position and $\theta_{1}$ is its measured position. The disturbing parameter $\delta$ has now to be estimated.

Let us consider the new control input $U^{*}$ taking into account the effect of the external disturbance. $U^{*}$ uses estimated pendulum position $\theta_{1}^{*}$ instead of the measured $\theta_{1}$ :

$$
U^{*}=i_{1}\left[\frac{\overline{m l} g}{i_{1}} \sin \left(\theta_{1}^{*}\right)-\ddot{\theta}_{1}^{r}+k_{p}\left(\theta_{1}^{*}-\theta_{1}^{r}\right)+k_{v}\left(\dot{\theta}_{1}^{*}-\dot{\theta}_{1}^{r}\right)\right]
$$

with $\dot{\theta}_{1}^{*}=\frac{d}{d t} \theta_{1}^{*}=\dot{\theta}_{1}+\frac{d}{d t} \delta$. Note that $\delta$ is constant resulting in $\frac{d}{d t} \delta=0$ therefore $\dot{\theta}_{1}^{*}=\dot{\theta}_{1}$. Injecting (5) in (6), while considering the preceding remark, results in:

$$
U^{*}=i_{1}\left[\frac{\overline{m l} g}{i_{1}} \sin \left(\theta_{1}+\delta\right)-\ddot{\theta}_{1}^{r}+k_{p}\left(\theta_{1}+\delta-\theta_{1}^{r}\right)+k_{v}\left(\dot{\theta}_{1}-\dot{\theta}_{1}^{r}\right)\right]
$$

Assume that $\delta$ is small, therefore sinus term in (6) can be linearized as follows:

$$
\sin \left(\theta_{1}+\delta\right) \approx \sin \theta_{1}+\delta \cos \theta_{1}
$$

The control input $U^{*}$ of the disturbed system can then be expressed in terms of the nominal control input $U$ and the parameter $\delta$ as:

$$
\begin{aligned}
U^{*} & =i_{1}\left[\frac{\overline{m l} g}{i_{1}}\left(\sin \theta_{1}+\delta \cos \theta_{1}\right)-\ddot{\theta}_{1}^{r}+k_{p}\left(\theta_{1}+\delta-\theta_{1}^{r}\right)+k_{v}\left(\dot{\theta}_{1}-\dot{\theta}_{1}^{r}\right)\right] \\
& =U+i_{1} k_{p} \delta+\overline{m l} g \delta \cos \theta_{1}
\end{aligned}
$$

The disturbed acceleration of the inertia wheel $\ddot{\theta}_{2}^{*}$ can then be expressed in terms of the nominal acceleration $\ddot{\theta}_{2}$. For that, injecting (9) in (2), and considering (4) leads to:

$$
\ddot{\theta}_{2}^{*}=\ddot{\theta}_{2}+\frac{i_{1}+i_{2}}{i_{1} i_{2}}\left(i_{1} k_{p} \delta+\overline{m l} g \delta \cos \theta_{1}\right)
$$

In order to express the velocity of the inertia wheel at the end of a cycle in terms of $\delta$, the integration of $\ddot{\theta}_{2}^{*}$ over one cycle gives:

$$
\begin{array}{r}
\dot{\theta}_{2}^{*}((k+1) \tau)-\dot{\theta}_{2}^{*}(k \tau)=\dot{\theta}_{2}((k+1) \tau)-\dot{\theta}_{2}(k \tau) \\
+\frac{i_{1}+i_{2}}{i_{2}} k_{p} \delta \tau+\frac{i_{1}+i_{2}}{i_{1} i_{2}} \overline{m l} g \delta \int_{k \tau}^{(k+1) \tau} \cos \theta_{1}(t) d t
\end{array}
$$

In the nominal case (without considering the persistant disturbance), since the optimization parameter of the reference trajectories is chosen in order to minimize the velocity of the inertia wheel at the end of a cycle, it can be assumed that $\dot{\theta}_{2}((k+1) \tau)=0$.

The estimation of the disturbed inertia wheel velocity at the end of each cycle is used in the computation of the control input used in the tracking of reference trajectories in the next cycle. In steady state $\dot{\theta}_{2}^{*}(k \tau)=\dot{\theta}_{2}(k \tau)$ so that the variation of the parameter $\delta$ for the next period can be computed. For that, substituting those values in (11) leads us to the following integral expression:

$$
\dot{\theta}_{2}^{*}((k+1) \tau)=\frac{i_{1}+i_{2}}{i_{2}}\left(k_{p} \delta \tau+\frac{\overline{m l} g \delta}{i_{1}} \int_{k \tau}^{(k+1) \tau} \cos \theta_{1}(t) d t\right)
$$

In order to use this relation between the inertia wheel rotation speed $\dot{\theta}_{2}^{*}$ and the parameter $\delta$, the integral of equation (12) containing $\theta_{1}$ must be expressed in terms of $\theta_{1}^{*}$. Consider (5), the linearized cosinus term gives:

$$
\cos \left(\theta_{1}\right) \approx \cos \theta_{1}^{*}+\delta \sin \theta_{1}^{*}
$$

Equation (13) in (12) leads to:

$$
\begin{aligned}
\frac{i_{2}}{i_{1}+i_{2}} \dot{\theta}_{2}^{*}((k+1) \tau) & =k_{p} \delta \tau+\frac{\overline{m l} g \delta}{i_{1}} \int_{k \tau}^{(k+1) \tau} \cos \theta_{1}^{*}(t) d t \\
& +\frac{\overline{m l} g \delta^{2}}{i_{1}} \int_{k \tau}^{(k+1) \tau} \sin \theta_{1}^{*}(t) d t
\end{aligned}
$$

Equation (14) can be seen as a second order polynomial in $\delta$ :

$$
a \delta^{2}+b \delta+c=0
$$


with the following coefficients:

$$
\begin{aligned}
a & =\frac{\overline{m l} g}{i_{1}} \int_{k \tau}^{(k+1) \tau} \sin \theta_{1}^{*}(t) d t \\
b & =k_{p} \tau+\frac{\overline{m l} g}{i_{1}} \int_{k \tau}^{(k+1) \tau} \cos \theta_{1}^{*}(t) d t \\
c & =-\dot{\theta}_{2}^{*}((k+1) \tau) \frac{i_{2}}{i_{1}+i_{2}}
\end{aligned}
$$

Roots of this polynomial can be computed in real time at the end of each period. The first root corresponds to the variation of $\delta$ which compensates the shift observed in the preceding cycle. At end of the $(k+1)^{t h}$ period, the parameter is updated in the following manner:

$$
\begin{aligned}
\delta_{k+1} & =\delta_{k}+\Delta \delta_{k+1} \\
\delta_{0} & =0
\end{aligned}
$$

The value $\Delta \delta_{k+1}$ is the computed root at the end of period $k$ given the observed value $\dot{\theta}_{2}^{*}((k+1) \tau)$ and assuming that the control law was computed during preceding period given adjusted value $\theta_{1}^{*}+\delta_{k}$.

\section{REAL-TIME EXPERIMENTS}

The extended control approach, proposed in this paper, has been implemented in real-time on the inertia wheel inverted pendulum testbed, described in section II. The dynamic parameters of the inverted pendulum are described in Table I. Parameters of the reference periodic trajectories and the feedback gains are summarized in Table II. Integrals within equation (14) are evaluated numerically. Two experimental scenarios are proposed, they correspond to two disturbance cases: (i) external disturbing added weight and (ii) a slope in the inclinometer fixation.

\section{A. Real-time experiment with a weight added to the pen- dulum body}

Consider the inertia wheel inverted pendulum with a disturbing weight fixed on the body of the pendulum

TABLE I

DESCRIPTION OF DYNAMIC PARAMETERS OF THE INERTIA WHEEL INVERTED PENDULUM

\begin{tabular}{clcc}
\hline Parameter & Description & Value & Unit \\
\hline \hline$m_{1}$ & body mass & 3.228 & $\mathrm{~kg}$ \\
\hline$m_{2}$ & wheel mass & 0.86422 & $\mathrm{~kg}$ \\
\hline$i_{1}$ & body inertia & $3.042 \times 10^{-2}$ & $\mathrm{~kg} \mathrm{~m}^{2}$ \\
\hline$i_{2}$ & wheel inertia & $7.986 \times 10^{-4}$ & $\mathrm{~kg} \mathrm{~m}^{2}$ \\
\hline$l_{1}$ & body CoM position & $6.354 \times 10^{-4}$ & $\mathrm{~m}$ \\
\hline$l_{2}$ & wheel CoM position & $52 \times 10^{-3}$ & $\mathrm{~m}$ \\
\hline
\end{tabular}

TABLE II

DESCRIPTION OF THE REFERENCE TRAJECTORIES AND CONTROL PARAMETERS

\begin{tabular}{clcc}
\hline Parameter & Description & Value & Unit \\
\hline \hline$A$ & oscillation amplitude & 3 & deg \\
\hline$\tau$ & oscillation period & 2 & $s$ \\
\hline$k_{p}$ & proportional gain & 300 & $1 / s^{2}$ \\
\hline$k_{v}$ & derivative gain & 20 & $1 / s$ \\
\hline
\end{tabular}

as shown in figure 4. This experiment of $60 \mathrm{sec}$ starts

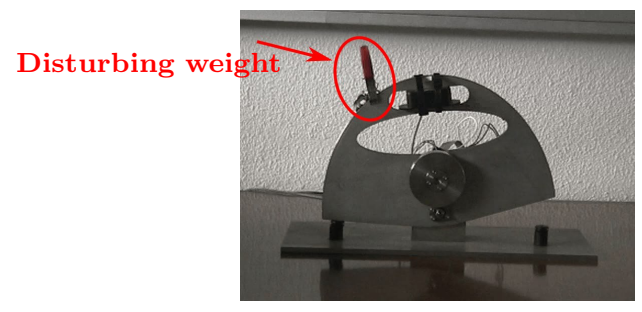

Fig. 4. Weight added as external persistant disturbance

with the disturbing weight fixed on the pendulum body. The weight is removed at about $30 \mathrm{sec}$. The weight assymetrically fixed to the pendulum body induces a torque acting on the pendulum axis as a disturbing external torque, resulting in a non-null value of $C_{1}$ in the dynamic model. Although the intensity of the induced torque varies during one period, it's effect over one period is constant since the trajectory of the pendulum is periodic. The induced disturbance is therefore seen as constant as its estimation is performed at the end of each period. The proposed method is then able to compensate such a disturbance. The obtained results are shown in Figures 5 to 10. The evolution of the angular position and velocity of the pendulum are presented in Figure 5 showing the convergence to a stable limit cycle corresponding to the reference periodic trajectories. This limit cycle is clearly shown in Figure 7 of the phase portrait $\left(\theta_{1}, \dot{\theta}_{1}\right)$. The evolution of the inertia wheel velocity is shown in Figure 6 , where the effect of the disturbing weight at the beginning and at $30 \mathrm{sec}$ can easily be seen. However the controller is able to reject the disturbance and steer the inertia wheel oscillating around the origin. Figure 8 displays the evolution of the control input. The normalized optimisation parameter is plotted in figure 9 . The estimated disturbance parameter $\delta$, plotted in figure 10, converges in about $30 \mathrm{sec}$ to an offset corresponding to an estimation of the disturbance to be compensated, after removing the disturbance it should converge to zero, however, due to the calibration of the inclinometer which is not absolutely perfect, the final estimated offset is slightly different from zero. A demonstration movie has been submitted accompanying the paper.

\section{B. Real time experments with a bad inclinometer calibra- tion}

In this experiment, the basic principle is to remove the $\theta_{1}$ position sensor (inclinometer) and to fix it again not straight but with an unknown slope. This will generate an offset in the measure of $\theta_{1}$ and the objective is to see whether the controller is able to deal with this persistant disturbance. The obtained results are displayed in Figures 11 to 16 . According to the obtained results, it can be seen that the controller is able to estimate the offset of the measure and to compensate it. 

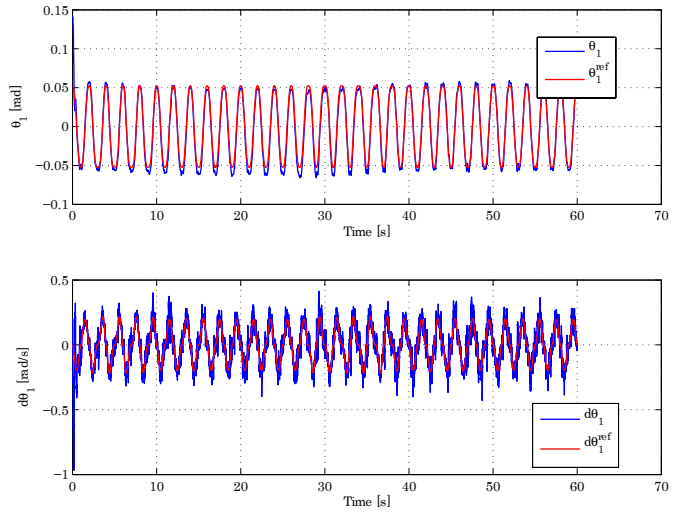

Fig. 5. Pendulum axis position and velocity

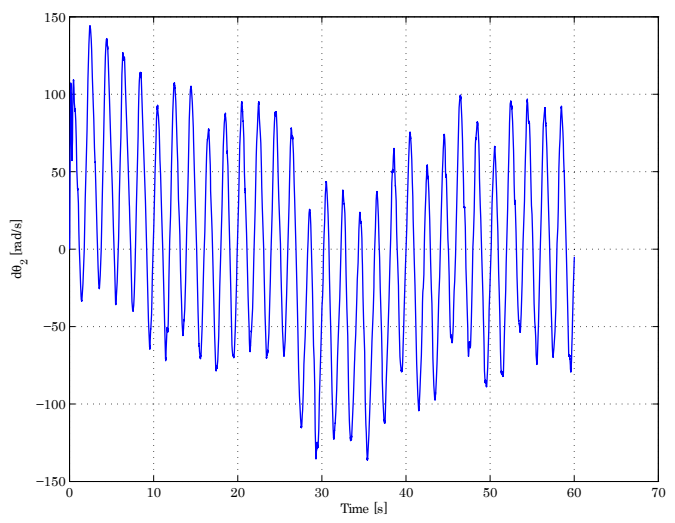

Fig. 6. Inertia wheel rotation speed

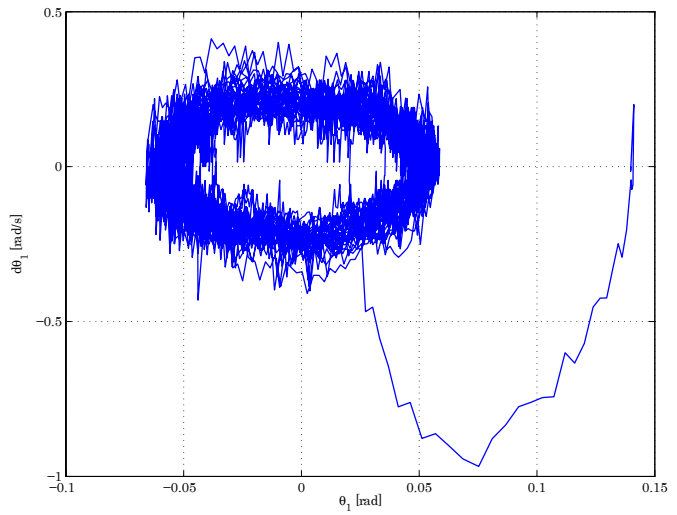

Fig. 7. Phase portrait $\left(\theta_{1}, \dot{\theta}_{1}\right)$

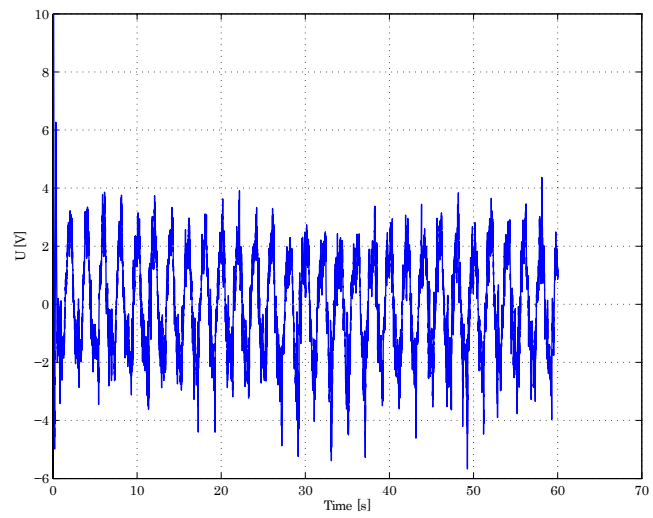

Fig. 8. Control input

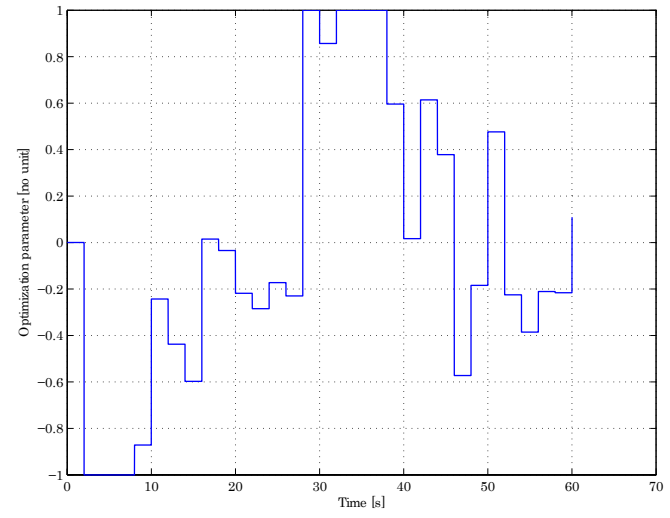

Fig. 9. Optimization parameter $p$

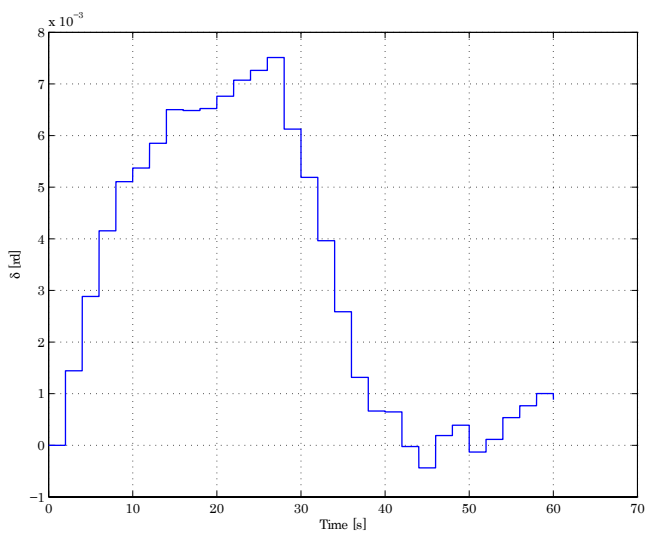

Fig. 10. Identified Parameter $\delta$
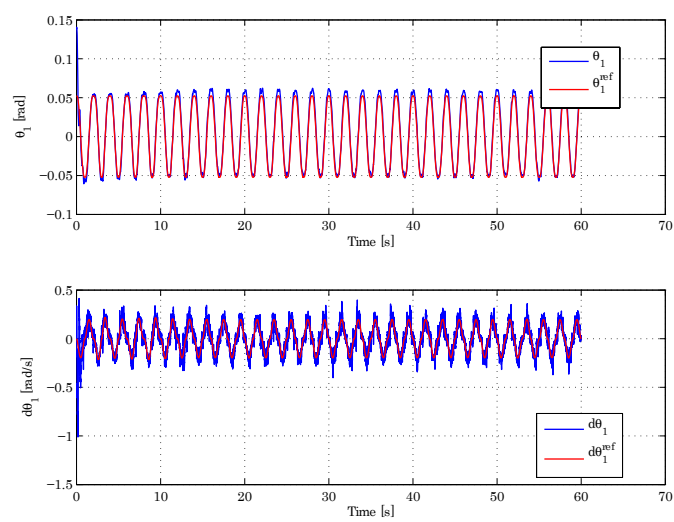

Fig. 11. Pendulum axis position and velocity

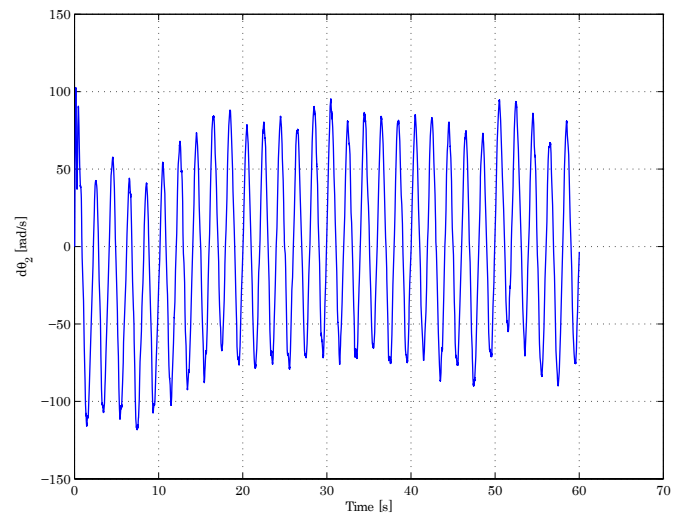

Fig. 12. Inertia wheel rotation speed 


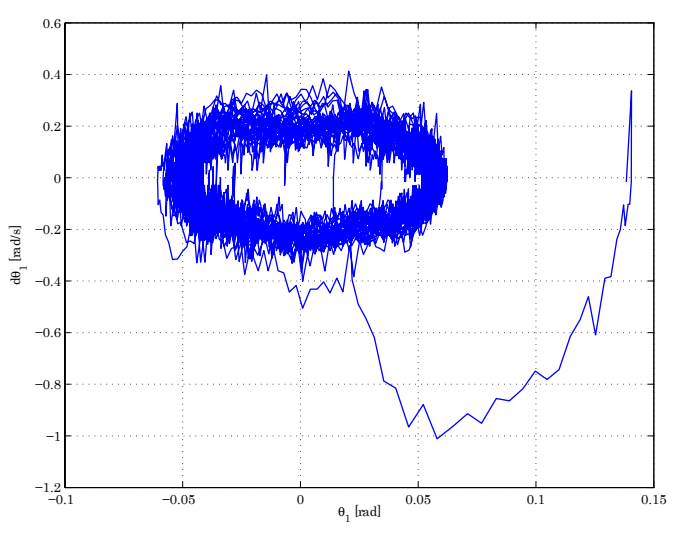

Fig. 13. Phase portrait $\left(\theta_{1}, \dot{\theta}_{1}\right)$

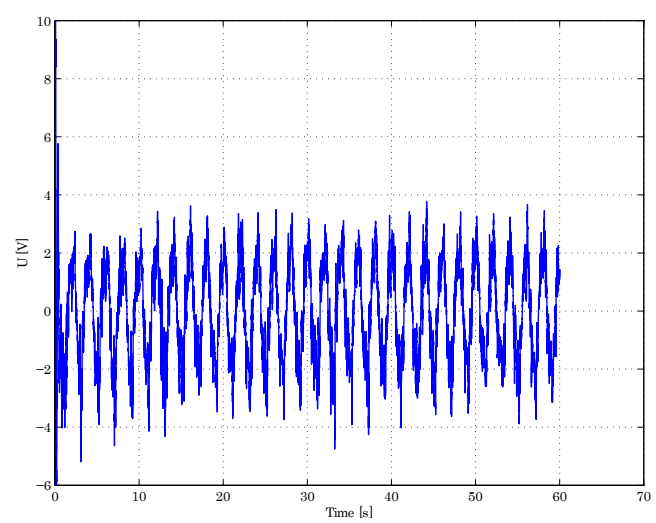

Fig. 14. Control input

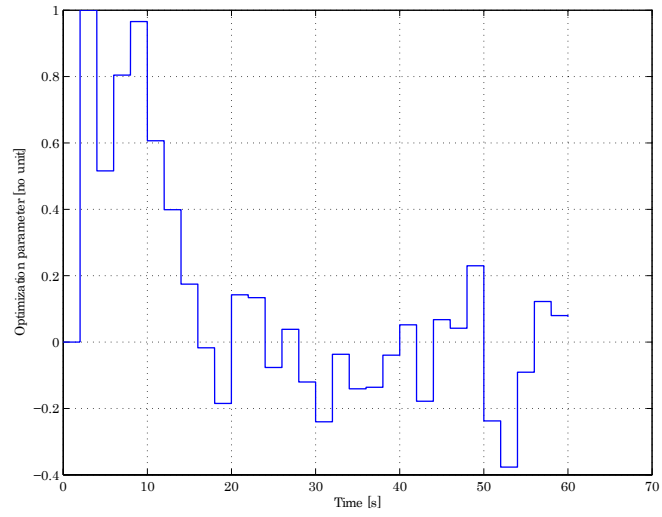

Fig. 15. Optimization parameter $p$

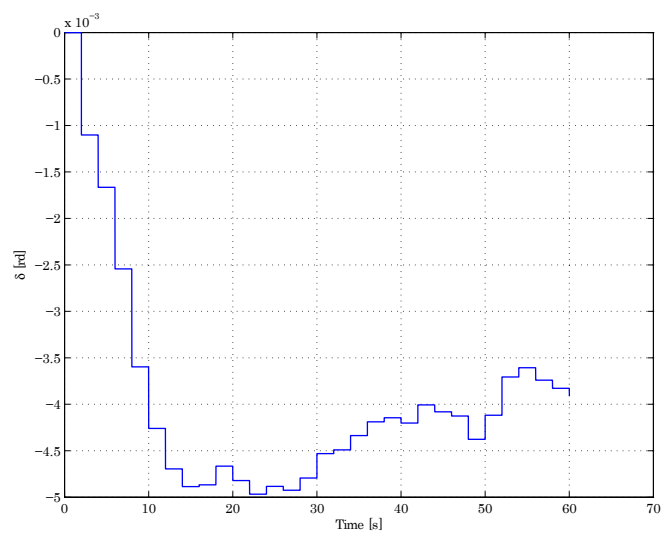

Fig. 16. Identified Parameter $\delta$

\section{Conclusion}

In this paper, the control approach proposed in [1] for stable limit cycle generation on underactuated mechanical systems has been extended in order to compensate constant disturbances which were not handled by the original method. The technique presented performs constant disturbance compensation, using online iterative estimation of an equivalent constant disturbance which should easily be compensated in the control loop. The technique showed its effectiveness in real time experiments on an underactuated robotics system, the inertia wheel inverted pendulum. Persistant disturbances such as a weight asymmetrically added to the pendulum body or sensor bad calibration were successfully compensated by the proposed controller.

\section{REFERENCES}

[1] Sébastien Andary, Ahmed Chemori, and Sébastien Krut. Stable Limit Cycle Generation for Underactuated Mechanical Systems, Application: Inertia Wheel Inverted Pendulum. In IROS'08: EEE/RSJ International Conference on Intelligent RObots and Systems, 2008.

[2] Juhoon Back and Hyungbo Shim. Adding robustness to nominal output-feedback controllers for uncertain nonlinear systems: A nonlinear version of disturbance observer. Automatica, 44(10):2528-2537, 2008.

[3] E. J. Davison. The Robust Control of a Servomechanism Problem for Time-Invariant Multivariable systems. IEEE transactions on Automatic Control, 21(2):25-34, 1976.

[4] Kwang Sik Eom, Il Hong Suh, Wan Kyun Chung, and SangRok Oh. Disturbance observer based force control of robot manipulator without force sensor. In ICRA, pages 3012-3017, 1998.

[5] B. A. Francis and W. M. Wonham. The Internal Model Principle of Control Theory. Automatica, 12(5-E):457-465, 1976.

[6] J. Huang. Asymptotic tracking and disturbance rejection in uncertain nonlinear systems. IEEE transactions on Automatic Control, 40(4):1118-1122, 1995

[7] A. Isidori. A remark on the problem of semiglobal nonlinear output regulation. IEEE transactions on Automatic Control, 42(12):1734- 1738, 1997.

[8] K. Ohnishi. A new servo method in mechatronics. Transactions of Japanese Society of Electrical Engineers, 107-D:83-86, 1987.

[9] Jung Rae Ryoo, Tae-Yong Doh, and Myung Jin Chung. Robust disturbance observer for the track-following control system of an optical disk drive. Control Engineering Practice, 12(5):577 - 585, 2004. Fuzzy System Applications in Control.

[10] L. Sciavicco and B. Siciliano. Modeling and control of robot manipulators. McGraw Hill, New York, 1996.

[11] M. Spong. Energy based control of a class of underactuated mechanical system. In Proc. 13th IFAC World Congress, volume $\mathrm{F}$, pages 431-436, 1996.

[12] M. Spong and M. Vidyasagar. Robot Dynamics and Control. John Wiley and Sons, 1989.

[13] M. Steinbuch. Repetitive control for systems with uncertain period-time. Automatica, 38(12):2103-2109, 2002.

[14] Z. Sun and T. Tsao. Rejectionof disturbance with nonlinear dynamics. In American Control Conference, pages 2573-2577, 1999. 